\title{
Advanced Fiber-Optic Acoustic Sensors
}

\author{
João G. V. TEIXEIRA ${ }^{1,2^{*}}$, Ivo T. LEITE², Susana SILVA², and Orlando FRAZÃO ${ }^{2,3}$ \\ ${ }^{1}$ EQS Group, Rua Joaquim Dias Rocha 354, 4470-221 Maia, Portugal \\ ${ }^{2}$ INESC TEC (coordinated by INESC Porto), Rua Campo Alegre 687, 4169-007 Porto, Portugal \\ ${ }^{3}$ Department of Physics and Astronomy, Faculty of Sciences - University of Porto, Rua Campo Alegre 687, 4169-007 \\ Porto, Portugal \\ "Corresponding author: João G. V. TEIXEIRAＥ-mail: joao.teixeira@eqs-global.com
}

\begin{abstract}
Acoustic sensing is nowadays a very demanding field which plays an important role in modern society, with applications spanning from structural health monitoring to medical imaging. Fiber-optics can bring many advantages to this field, and fiber-optic acoustic sensors show already performance levels capable of competing with the standard sensors based on piezoelectric transducers. This review presents the recent advances in the field of fiber-optic dynamic strain sensing, particularly for acoustic detection. Three dominant technologies are identified - fiber Bragg gratings, interferometric Mach-Zehnder, and Fabry-Pérot configurations - and their recent developments are summarized.
\end{abstract}

Keywords: Acoustic sensors, fiber optics, optical fiber sensors

Citation: João G. V. TEIXEIRA, Ivo T. LEITE, Susana SILVA, and Orlando FRAZÃO, "Advanced Fiber-Optic Acoustic Sensors," Photonic Sensors, 2014, 4(3): 198-208.

\section{Introduction}

Acoustic wave detection and analysis is a field of research with a vast number of applications which have grown a lot in the past few decades. Acoustic methods have proved to be very informative tools for studying the structure and properties of materials and different physical processes occurring inside them. Thus, the importance of the acoustic wave technology on both modern science and engineering applications, has been growing at the increasing speed. The applications spread to diverse areas such as navigation (pressure and speed monitoring, depth sounding/sea-bottom profiling), non-destructive evaluation of structures through detection of emitted ultrasounds (detection and location of cracks, inner stresses, micro displacements, and others), detection of phase transitions in scientific research, as well as in the health sciences (medical imaging and diagnosis). All these applications have the acoustic sensor as the core instrument to capture the acoustic fields from which all data are extracted. Apart from audio-used microphones, the most common and commercially available acoustic sensors are based on piezoelectric crystals. Over the years, piezoelectric sensors have been used in many acoustic applications with a fair amount of success. They are small in size, relatively inexpensive, quite sensitive, and capable of measuring a wide variety of different input parameters. Nevertheless, they do exhibit some limitations, especially when being submitted to harsh environments, having low resistance to chemical agents and high temperatures,

Received: 8 October 2013/ Revised version: 2 April 2014

(C) The Author(s) 2014. This article is published with open access at Springerlink.com

DOI: $10.1007 / \mathrm{s} 13320-014-0148-5$

Article type: Review 
and they are not immune to electromagnetic interference. This type of system also needs many electronic components and electric cable that, together with the high attenuation of electronic signals, makes them less practical in many applications where it is necessary to cover great distances (e.g. remote sensing or long structures). Fiber-optic sensors remove all these limitations from acoustic sensing and bring more advantages to it. Fiber-optic systems have the large bandwidth, are not constrained to the limited size and shape, are robust lasting solutions, capable of multiplexing, and can be directly connected to optical transmission links. These features make fiber-optics a very promising technology for acoustic sensors. The first systems were reported in 1977 by Bucaro et al. and Cole et al. [1-3]. Their preliminary works marked the beginning of fiber-optic acoustic sensing that, since then, continued to be developed. Currently, numerous works in this field can be found, many of these following interferometric configurations such as Mach-Zehnder [4], Michelson [5], Sagnac [6], and Fabry-Pérot [7], while others explore the possibilities offered by fiber Bragg grating (FBG) sensors [8]. A comprehensive review paper in this area until 2008 was written by Graham et al. [9], and another work comparing three different types of fiber-optic acoustic sensors was published in 2010 [10]. This review summarizes these sensing technologies, with particular emphasis on works reported recently in the field of advanced fiber-optic acoustic sensing.

\section{Interferometric acoustic sensors}

\subsection{Mach-Zehnder configuration}

As it was pointed out in the introduction, the first work in the field of fiber-optic acoustic sensing was done by Bucaro et al. $[1,2]$. Their detection method consisted of a fiber-optic Mach-Zehnder interferometer (MZI). By subjecting one arm of the fiber interferometer to an acoustic wave, the authors showed that the acoustic wave interaction with the fiber leads to a variation in the optical path length. Therefore, the characteristics of the acoustic wave can be deduced through the observed changes in the intensity of the interferometer output. This interferometer worked with sound frequencies of $40 \mathrm{kHz}-400 \mathrm{kHz}$ with no significant sensitivity variation. The fiber MZI was used in many assemblies to further study the capabilities of fiber optic acoustic sensors and, in particular, to create optical fiber hydrophones $[2,11]$. Another important work using a fiber MZI setup was demonstrated in 1990 [12], where the configuration was based on the work path of Hocker in sensitivity enhancement by coating the fiber with composite structures of low elastic modulus [13, 14]. It was shown that, by adding a piezoactive polymer coating to the fiber, it was possible to enhance the sensitivity to the acoustic wave. A typical MZI configuration is illustrated in Fig. 1, where the sensing arm of the interferometer incorporates a fiber mandrel as a means of increasing the effective area of interaction with the acoustic wave.

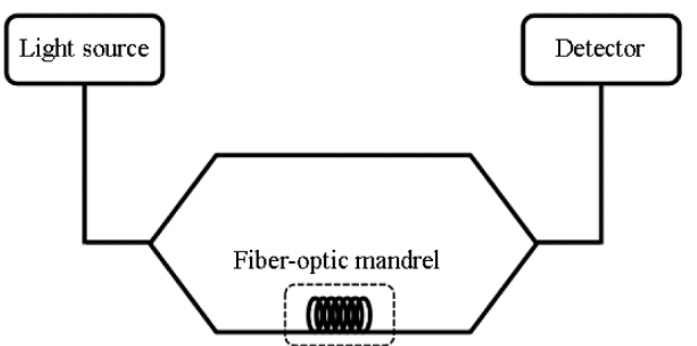

Fig. 1 Reflective spectrum of the hybrid fiber-optic Fabry-Pérot sensor.

\subsection{Michelson configuration}

Fiber-optic Michelson interferometers are also used in the context of acoustic detection, since it was demonstrated for the first time in $1980[15,16]$. The Michelson configuration consists of an optical fiber coupler where two output arms are used as reference and sensor paths. At the end of each arm, there is a reflecting structure (e.g. a mirror or an FBG) to send the two signals back to the detector. 
One can think of a Michelson setup (see Fig. 2) as half of a Mach-Zehnder, which makes its operation principle very similar. Although in this case, instead of a segment of the fiber being the detector, it is also possible that it is the reflecting structure acting as the acoustic transducer, responsible for the optical path difference. Michelson setups are probably the least used configuration for fiber-optic acoustic sensing.

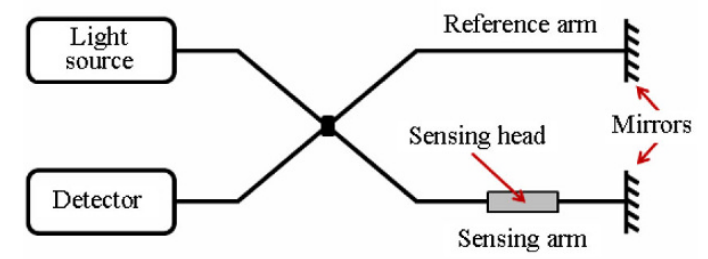

Fig. 2 Michelson fiber-optic interferometric configuration, consisting of a $2 \times 2$ coupler with 50:50 split, and with reflective elements (e.g. metallic mirror, FBGs, or other) added to the interferometer arms.

\subsection{Sagnac configuration}

The fiber-optic Sagnac interferometer is also one of the most explored configurations for optical acoustic sensing [6, 17]. This interferometer has been widely applied in the manufacture of fiber-optic hydrophone systems for acoustic detection. Figure 3(a) presents a typical configuration of the fiber-optic Sagnac interferometer. The final amplitude variation is a function of the hydrophone phase modulation and the product of the acoustic modulation frequency with the loop time delay. This differs from MZI sensors, where the final amplitude is the function only of the hydrophone phase modulation. The loop will have a proper frequency, which is the lowest frequency where the maximum sensitivity is achieved. This frequency is determined by the length of the delay loop.

A means of decreasing the fiber length used in each sensor is required, in order to use Sagnac-based sensors in large-scale arrays. In 1999, the first Sagnac in the array configuration was demonstrated [18]. In this case, the same delay coil was used for several parallel hydrophones, creating an array of sensors through time domain separation [see Fig. $3(b)]$.

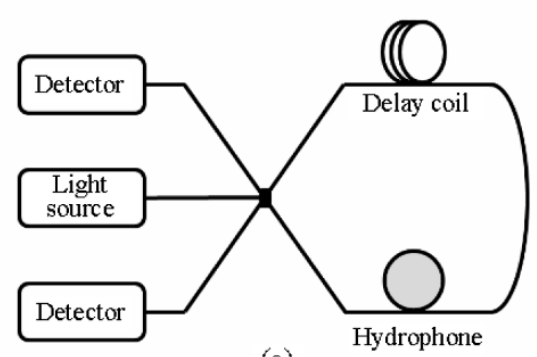

(a)

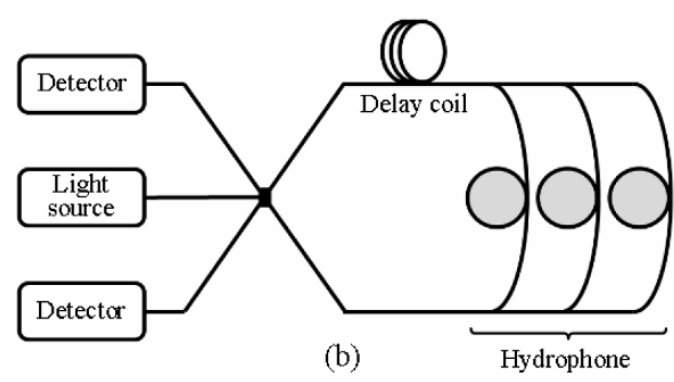

Fig. 3 Sagnac-based interferometric acoustic fiber-optic sensor in the (a) single-sensor configuration and (b) array sensor configuration.

\subsection{Fabry-Pérot configuration}

The interest in Fabry-Pérot interferometers (FPIs) to create fiber-optic acoustic sensors has grown over time, and it is now one solution being widely explored [7]. This interferometer gained relevance in the field of acoustic sensing with its demonstration in 1991 by Murphy and co-workers [19]. A strain sensitivity of 5.45 -degree phase shift/microstrain $\cdot \mathrm{cm}^{-1}$ was achieved. The extrinsic Fabry-Pérot (FP) cavity consisted of an air gap between a single-mode fiber and a multimode fiber, all inside in a hollow-core fiber, as illustrated in Fig.4(a). Variations in the air gap longitudinal dimension, due to the applied strains, modify the FP cavity characteristics.

Changes in the cavity can be deduced by analyzing the reflected wavelength variation, and therefore, the strain arising from the acoustic wave can be determined. There are many demonstrations of extrinsic FPIs for pressure and acoustic sensing using optical fibers [20-26]. A summary of these works reported up to 2011 can be found in [27]. 
Since Murphy's work, new solutions to increase the dynamic range and high pressure variation have been proposed. One of them is the FPI based on a diaphragm topology, as illustrated in Fig. 4(b). Diaphragms of different materials with different thicknesses may present the enhanced sensitivity to acoustic waves.

(a)

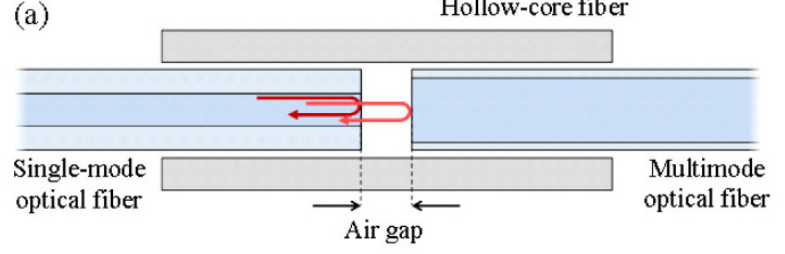

(b)

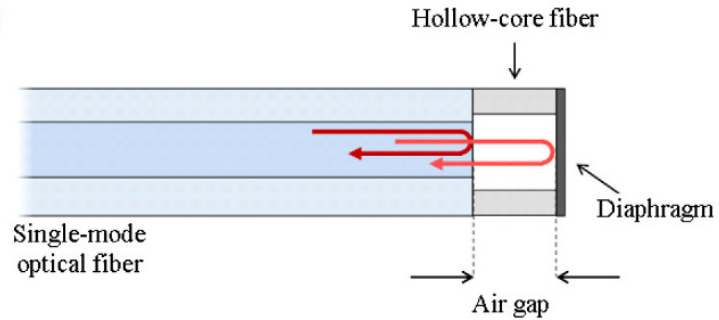

Fig. 4 Extrinsic Fabry-Pérot interferometer: (a) fiber air gap based cavity and (b) diaphragm based cavity.

A review of interferometric fiber-optic acoustic sensors until 2004 can be found in [28]. Another review of the developments up to 2012 of interferometric fiber optic sensors is given by Lee et al. [29].

\section{Fiber Bragg gratings}

The optical FBG was first demonstrated by Hill et al. in 1978 [30]. As shown in Fig. 5, the FBG is a periodic microstructure printed by means of a perturbation of the refractive index of the fiber core. The structure will reflect only a specific wavelength, which depends on the periodic spacing of the FBG. By analyzing the shifts in the reflected wavelength, the spacing variation can be determined. Exploring this effect, many sensors can be built based on the FBG principle. The most commonly used are FBG-based strain and temperature fiber-optic sensors.

In 1996, Webb et al. were the first to apply FBGs to sensing of ultrasonic fields for medical applications [31]. As the acquisition rate of wavelength interrogators increased, the first dynamic study appeared. Betz et al. used FBGs for the monitoring and detection of Lamb waves [32-34], while other groups investigated the possibility of using FBGs for dynamic strain sensing [35-37]. In 2009, Seo et al. studied the sensitivity enhancement of FBG sensors for acoustic emission detection [38]. The FBG was compared with typical acoustic emission piezoelectric sensors and demonstrated close performance, with similar waveforms, when subjected to pencil lead breaks. One of the great advantages of FBGs over optical fiber interferometers is their ease of multiplexing. FBGs also show improved spectral characteristics but their drawback is their sensitivity. There are at least two ways of achieving the improvement of the FBG sensitivity. One is by reducing the radius of the fiber and, consequently, of the FBG itself. However, this will lead to a lower mechanical resistance of both the sensor and the fiber. The other is by analyzing simultaneously the reflected and transmitted optical signals, instead of just one of them, using the so-called transmit reflect detection sensing (TRDS) interrogation technique. When the acoustic waves interact with the FBG, the reflected wavelength undergoes a shift, either positive or negative, while the transmitted signal experiences an opposite shift; hence, the overall signal can be increased by differential amplification. The TRDS system is well described in a review of FBG-based sensors for acoustic detection [39].

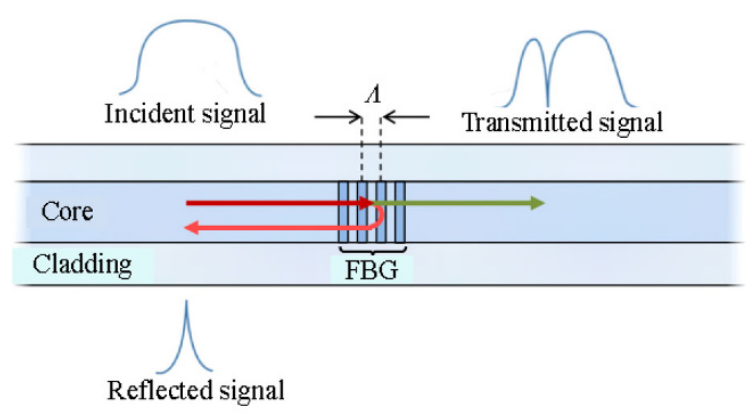

Fig. 5 Operation principle of an FBG based optical sensor (the reflected Bragg wavelength $\lambda_{B}$ is related to the grating period $\Lambda$ of the FBG through $\lambda_{B}=2 n_{e} \Lambda$, where $n_{e}$ is the effective refractive index of the structure). 


\section{Advanced technologies}

\subsection{Compact directional acoustic sensor using a multi-fiber optical probe}

Bucaro et al. created a compact directional acoustic sensor [40] based on a previous work [41], the microphone, which in turn was based on an earlier reported fiber-lever probe [42]. This sensor is composed of a two-fiber optical probe, an optical source, a photo-detector, and a slender cylindrical cantilever to which an optical reflector is attached, as depicted in Fig. 6(a). While one fiber is used for illuminating the cantilever, the other collects the reflected optical signal and is responsible for the directionality sensitivity. Directionality is achieved through the fact that the power in the collection fiber varies proportionally to the cosine of the angle between the axis of the two fibers [ $x$ axis in Fig. $6(\mathrm{~b})]$ and the cantilever tip displacement direction [in the $x-y$ plane in Fig. 6(b)] which, in turn, is in the same direction as the acoustic wave. This type of sensor requires only about $100-\mathrm{mW}$ electrical power and has a low level of $1 / f$ noise. This sensor can be a solution for applications where a relatively long battery powered operational life time is required.

\subsection{Enhancement of acoustic sensitivity of hollow-core photonic bandgap fibers}

Yan et al. reported the enhancement of the acoustic sensitivity with hollow-core photonic bandgap fibers (HC-PBFs) by using air-included polymer coatings [43]. The first investigations in this field were performed by Cole et al., where it was demonstrated that this type of coated fiber has similar normalized responsivity (NR) to the mandrel based hydrophones [44, 45]. The developed HC-PBF was coated with a $7-\mu \mathrm{m}$-thickness silica cladding and a 100- $\mu \mathrm{m}$-thickness Parylene-C polymer, and exhibited a pressure sensitivity of $10 \mathrm{~dB}$, which is higher than that of the commercial HC-1550-02 fiber, and $25 \mathrm{~dB}$ higher than that of standard single-mode fibers. Many air columns along the fiber significantly reduced its effective Young's modulus and increased the axial strain due to the acoustic pressure, resulting in a higher sensitivity. The acoustic sensitivity could be further enhanced by optimizing the micro-structured cladding and the polymer coating. This significant sensitivity improvement can simplify the design of fiber hydrophone arrays and also increase the number of sensors that could be multiplexed in a single fiber.

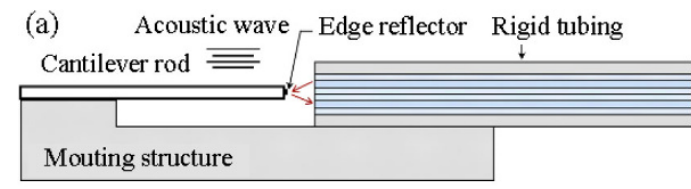

(b)

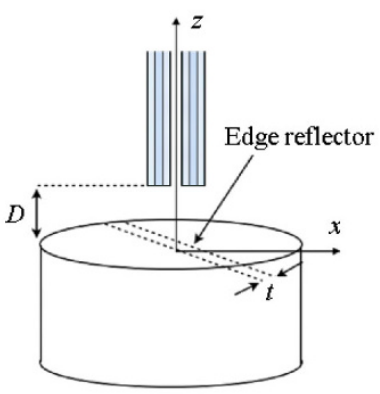

Fig. 6 Sensing configuration demonstrated in [40] for a two-fiber optical probe with the cantilever for acoustic wave detection.

\subsection{Fiber laser sensor based on a phase-shifted chirped grating for acoustic sensing of partial discharges}

Lima et al. demonstrated a fiber laser sensor based on a phase-shifted chirped FBG (PS-CFBG) for acoustic sensing of partial discharges [46]. The PS-CFBG sensing head had a narrow passband peak with a bandwidth of $20 \mathrm{pm}$ at $-3 \mathrm{~dB}$. Since a relatively flat frequency response in the range of $50 \mathrm{kHz}-200 \mathrm{kHz}$ was desirable, a mandrel was used to increase the interaction area between the fiber and the incident pressure wave. The mandrel consisted in a 20-mm-length cylinder of polycarbonate, with an outer diameter of $10 \mathrm{~mm}$ and a thickness of $0.5 \mathrm{~mm}$. In the reported results, the worst acoustic receiving sensitivity for the sensor was $-200 \mathrm{~dB}$ (@) $0 \mathrm{~dB}=$ $1 \mathrm{~V}_{\mathrm{rms}} / \mu \mathrm{Pa}$ ) at the frequency of $250 \mathrm{kHz}$, which 
allowed a pressure resolution of about $10 \mathrm{~Pa}$. The results also showed that this kind of optical solution for acoustic sensing of partial discharges presents an enhanced sensitivity and superior performance when compared with the piezoelectric transducer (PZT) sensors, which have to be mounted externally on the structure. The fact that the optical sensor can be mounted on the inside wall, is an inherent advantage allowing for better sensitivity and location processes. Another work on high sensitivity phase-shifted FBGs was developed by Azmi et al. where a $20-\mathrm{dB}$ sensitivity enhancement compared with standard FBG acoustic sensors was reported [47]. This would be later applied to failure monitoring of e-glass/vinylester composite structures [48].

\subsection{Highly sensitive fiber pressure sensors based on off-center diaphragm reflection}

Qi et al. developed a fiber collimator diaphragm pressure sensor, where the detection mechanism is based on an incidence-angle sensitivity instead of the traditional working-distance sensitivity [49]. This configuration is schematically illustrated in Fig. 7. The high sensitivity is attained due to coupling efficiency of the collimator, which is very sensitive to the incidence angle of the off-center diaphragm reflection. Another particular feature of this sensor is that the diaphragm cavity is sealed and pressurized. In this way, the displacement of the diaphragm is determined by the pressure difference between the

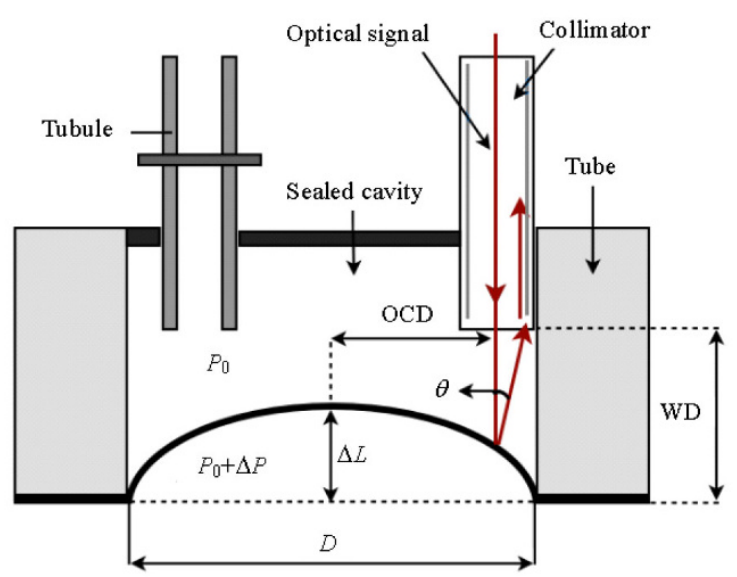

Fig. 7 Sensing configuration based on a fiber collimator and off-center reflection on a diaphragm demonstrated in [49]. inside and outside, making it possible to tune the detection range by changing the pressure inside the sealed diaphragm cavity. Results showed sensitivities of $1.11 \mathrm{~dB} / \mathrm{kPa}$ and $0.16 \mathrm{~dB} / \mathrm{kPa}$, for silicon diaphragm thicknesses of $100 \mu \mathrm{m}$ and 150 $\mu \mathrm{m}$, respectively.

\subsection{Fiber-optic Fabry-Pérot acoustic sensor with multilayer graphene diaphragm}

Ma et al. have developed diaphragm-based FP acoustic sensors with the ultra-thin multilayer graphene diaphragms [22], as shown in Fig. 8. This can be regarded as an extension to dynamic measurement of their previously reported pressure sensors, where a diaphragm with the thicknesses of about $0.71 \mathrm{~nm}$ and $25 \mu \mathrm{m}$ in diameter was tested, achieving a pressure sensitivity (pressure induced diaphragm reflection) of $39.4 \mathrm{~nm} / \mathrm{kPa}$ [21]. Graphene structures have the very high mechanical strength, and it is possible to build ultra-thin diaphragms capable of withstanding high pressures. The possibility of making very thin diaphragm structures can significantly improve the pressure sensitivity of FP sensors as demonstrated in [21]. This acoustic sensor had a multilayer graphene diaphragm with the thickness of $100 \mathrm{~nm}$ and diameter of $125 \mu \mathrm{m}$. The dynamic (acoustic) pressure tests showed that the sensor had a pressure sensitivity up to $1100 \mathrm{~nm} / \mathrm{kPa}$ and was able to detect acoustic signals as small as $60 \mu \mathrm{Pa} / \sqrt{ } \mathrm{Hz}$ at $10 \mathrm{kHz}$, with a relatively uniform frequency response from $0.2 \mathrm{kHz}$ to $22 \mathrm{kHz}$. Decreasing the diaphragm thickness leads to a sensitivity improvement but with a detection range reduction.

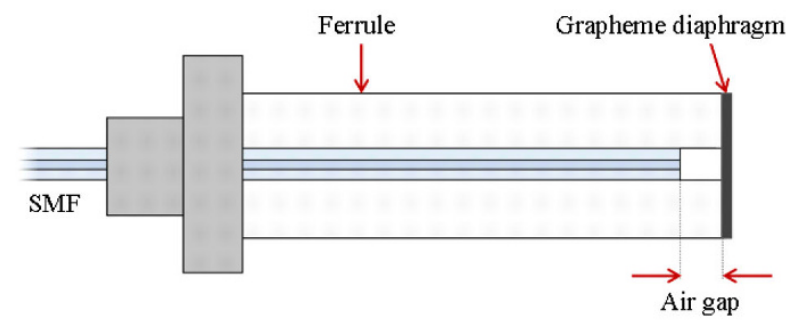

Fig. 8 Extrinsic Fabry-Pérot interferometer incorporating a multilayer graphene diaphragm (adapted from [22]). 
Recently, $\mathrm{Xu}$ et al. reported a fiber-tip FP interferometer made with a 125 - $\mu \mathrm{m}$-diameter and 130-nm-thickness silver diaphragm, demonstrating a pressure sensitivity of $70.5 \mathrm{~nm} / \mathrm{ka}$ [20].

\subsection{Miniature fiber acoustic sensors and sensor array using photonic-crystal membranes}

Digonnet et al. reported on the second generation of FPI acoustic sensors incorporating diaphragms made from highly reflective photonic crystals [51] as a microphone [50]. The sensitivity, reproducibility in the interrogation wavelength, and thermal stability were significantly enhanced by improving the design and choice of materials, and developing a more precise assembly process. The sensor was made of a highly reflective photonic crystal fabricated on a silicon diaphragm and a thin layer of gold evaporated on the cleaved tip of a single mode fiber. The thermal stability was achieved by attaching the diaphragm to the fiber through a housing made of silica with a very low thermal expansion coefficient. The housing connected the FP cavity to a large back chamber that eliminated the sensitivity to static pressure. The proposed structure is illustrated in Fig. 9. From measurements made with 16 sensors, the minimum detectable pressure in the flat band of 10 of them lies in $\pm 0.3 \mathrm{~dB}$ of a mean value of $50 \mu \mathrm{Pa} / \sqrt{\mathrm{Hz}}$. At the sensor's resonance frequency $(12.5 \mathrm{kHz})$, the

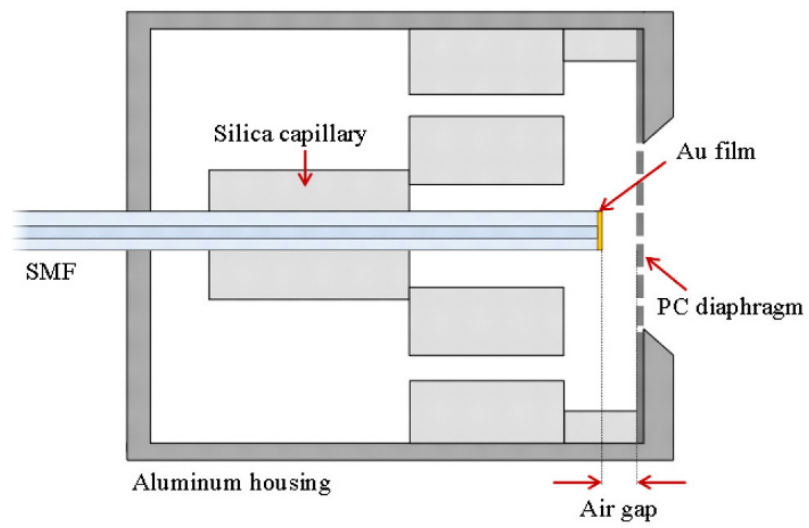

Fig. 9 Extrinsic Fabry-Pérot interferometer incorporating a highly-reflective photonic-crystal diaphragm and $\mathrm{Au}$ film evaporated on the cleaved tip of a single-mode optical fiber (adapted from [50]). mean value was $6.25 \mu \mathrm{Pa} / \sqrt{ } \mathrm{Hz}$. Thermal stability tests showed that the temperature could change as much as $68{ }^{\circ} \mathrm{C}$ before the acoustic sensitivity varied by more than $\pm 0.3 \mathrm{~dB}$. A configuration also consisting of an FPI incorporating a photonic crystal reflector was done by Kilic et al. [11]. The reported sensor was tested from $100 \mathrm{~Hz}$ to $100 \mathrm{kHz}$, demonstrating a sound-pressure-equivalent noise spectral density down to $12 \mu \mathrm{Pa} / \sqrt{ } \mathrm{Hz}$.

\subsection{Sensitive acoustic vibration sensor using single-mode fiber tapers}

Yi Li et al. followed the line of the previous work reported by Chen et al. [53] and presented a sensitive acoustic vibration sensor with a fiber taper as the sensing arm of an MZI setup [52], as shown in Fig. 10. The biconical fiber tapers were manufactured by elongating a single mode fiber heated by a hydrogen gas flame, and diameters ranging from $1.7 \mu \mathrm{m}$ to $50 \mu \mathrm{m}$ were achieved. Under the same conditions, the thinnest fiber taper showed a $20-\mathrm{dB}$ improvement in the signal-to-noise ratio compared with the standard single mode fibers. The taper waist length can still be further increased and made thinner, which should cause longer phase change when subjected to vibrations. In addition, the low refractive index polymer gel used as the bonding agent in the fabrication process also attenuates the loss in the acoustic transmission process.

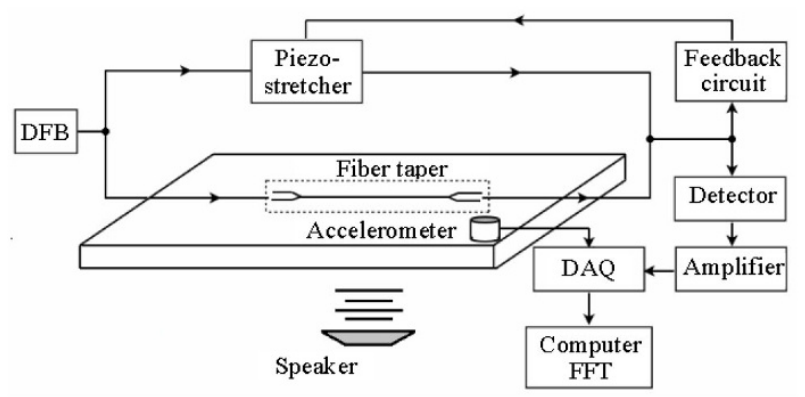

Fig. 10 Acoustic sensor based on a Mach-Zehnder interferometric configuration where a tapered segment of a single-mode optical fiber is placed in the sensing arm of the interferometer (adapted from [52]). 


\subsection{A vibration sensor based on a distributed Bragg reflector fiber laser}

M. S. Ferreira et al. demonstrated recently a vibration sensor based on a distributed Bragg reflector (DBR) fiber laser with a response up to about $1.5 \mathrm{kHz}$ [54]. The DBR sensing head was made of a linear laser cavity formed by two FBG gratings $20 \mathrm{~cm}$ apart from each other, written directly in a highly-doped $\mathrm{Er}^{3+}$-doped fiber [55]. The proposed configuration is schematically illustrated in Fig. 11. One of the FBGs was subjected to vibration using a PZT transducer (FBG2), while the other was used as a spectral filter (FBG1). The FBGs were fabricated so that the Bragg wavelengths would differ by $1 \mathrm{~nm}$. With this mismatch, no lasing occurred initially. However, when applying strain to FBG1, its Bragg wavelength could be tuned to match that of FBG2, allowing laser emission to occur. It was demonstrated that if the first grating is placed in the slope of the second one, not only the optical power increases, but also a higher sensitivity to frequency changes on the second grating is obtained.

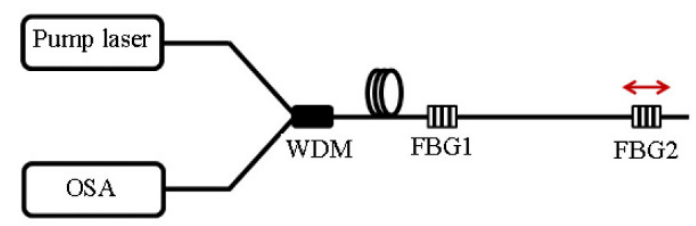

Fig. 11 Sensing configuration based on a DBR fiber laser (adapted from [54]).

In Table 1, we summarize the performance, in terms of resolution and frequency bandwidth, of the fiber-optic acoustic sensing technologies presented in this section.

Table 1 Performance of state-of-art acoustic sensors.

\begin{tabular}{cccc}
\hline Reference & Technology & Resolution & Freq. Range \\
\hline$[40]$ & N-I & $6 \mathrm{mPa} / \sqrt{ } \mathrm{Hz}$ & $50 \mathrm{~Hz}-1 \mathrm{kHz}$ \\
{$[49]$} & N-I & $1.11 \mathrm{~dB} / \mathrm{kPa}$ & $\mathrm{n} / \mathrm{a}$ \\
{$[22]$} & FPI & $60 \mu \mathrm{Pa} / \sqrt{H z}$ & $0.2 \mathrm{kHz}-22 \mathrm{kHz}$ \\
{$[50]$} & FPI & $50 \mu \mathrm{Pa} / \sqrt{ } \mathrm{Hz}$ & $2 \mathrm{kHz}-8 \mathrm{kHz}$ \\
{$[11]$} & FPI & $12 \mu \mathrm{Pa} / \sqrt{\mathrm{Hz}}$ & $0.1 \mathrm{kHz}-100 \mathrm{kHz}$ \\
{$[52]$} & MZI & $\mathrm{n} / \mathrm{a}$ & $30 \mathrm{~Hz}-40 \mathrm{kHz}$ \\
{$[54]$} & FBG & $\mathrm{n} / \mathrm{a}$ & $0-1.5 \mathrm{kHz}$ \\
\hline
\end{tabular}

N-I: non-interferometric.

\section{Conclusions}

We have given a review on the recent advances in fiber-optic methods for dynamic strain sensing, particularly in the acoustic and ultrasonic regions. Three major configurations have emerged among the others and form the present basis of research in the field: Mach-Zehnder/Sagnac interferometry, Fabry-Pérot interferometry, and fiber Bragg gratings. These technologies exhibit different merits, and the choice depends ultimately on the requirements of the application intended. The MZI/Sagnac interferometers are the most cost-effective and easier to implement. Also, the recent research is replacing the fiber mandrels by segments of tapered and hollow-core fibers, reducing the size of the sensing head. The most sensitive fiber-optic acoustic sensors reported so far are based on FP cavities, and the recent works suggest that this can be further improved by using diaphragms of different materials with the increased stiffness and lower thickness-to-diameter ratio. FBGs are most suitable for multiplexing, since wavelength multiplexing is easier to implement than time-domain multiplexing. Although this technology typically exhibits lower sensitivities, the recent developments (e.g. exploring phase-shifted gratings) are overcoming this drawback. Another trend being followed in fiber-optic acoustic sensing is the technique combining fiber-optics with micro-mechanical devices (e.g. cantilevers), where the acoustic wave does not interact directly with the optical fiber, but rather with an external mechanical element which modulates the light traveling in the fiber.

Fiber-optic acoustic sensors are now starting to compete with the standardly used PZT sensors in terms of the frequency range, resolution, and sensitivity. Further developments are expected in the three main technologies presented, with the application of specialty fibers and novel materials. 


\section{Acknowledgment}

This work is funded by the ERDF - European Regional Development Fund through the COMPETE Program (operational program for competitiveness) and by National Funds through the FCT - Fundação para a Ciência e a Tecnologia (Portuguese Foundation for Science and Technology) within project FCOMP - 01-0124-FEDER-022701.

Open Access This article is distributed under the terms of the Creative Commons Attribution License which permits any use, distribution, and reproduction in any medium, provided the original author(s) and source are credited.

\section{References}

[1] J. A. Bucaro, H. D. Dardy, and E. F. Carome, "Optical fiber acoustic sensor," Applied Optics, 16(7): 1761-1762, 1977.

[2] J. A. Bucaro, H. D. Dardy, and E. F. Carome, "Fiber-optic hydrophone," Journal of the Acoustical Society of America, 62(5): 1302-1304, 1977.

[3] J. H. Cole, R. L. Johnson, and P. G. Bhuta, "Fiber-optic detection of sound," Journal of the Acoustical Society of America, 62(5): 1136-1138, 1977.

[4] A. I. Hernández-Serrano, G. Salceda-Delgado, D. Moreno-Hernández, A. Martínez-Ríos, and D. Monzón-Hernández, "Robust optical fiber bending sensor to measure frequency of vibration," Optics and Lasers in Engineering, 51(9): 1102-1105, 2013.

[5] X. Hong, J. Wu, C. Zuo, F. Liu, H. Guo, and K. Xu, "Dual Michelson interferometers for distributed vibration detection," Applied Optics, 50(22): 4333-4338, 2011.

[6] J. L. Rivera, M. P. Sánchez, A. Miridonov, and S. Stepanov, "Adaptive Sagnac interferometer with dynamic population grating in saturable rare-earth-doped fiber," Optics Express, 21(4): 4280-4290, 2013.

[7] S. E. U. Lima, O. Frazão, F. M. Araújo, L. A. Ferreira, V. Miranda, and J. L. Santos, "Extrinsic and intrinsic fiber optic interferometric sensors for acoustic detection in high-voltage environments," Optical Engineering, 48(2): 024401, 2009.

[8] A. Wada, S. Tanaka, and N. Takahashi, "Optical fiber vibration sensor using FBG Fabry-Pérot interferometer with wavelength scanning and Fourier analysis," IEEE Journal Sensors, 12(1): 225-339,
2012.

[9] G. Wild and S. Hinckley, "Acousto-ultrasonic optical fiber sensors: overview and state-of-the-art," IEEE Journal Sensors, 8(7): 1184-1193, 2008.

[10] S. E. U. Lima, O. Frazão, R. G. Farias, F. M. Araújo, L. A. Ferreira, J. L. Santos, et al., "Mandrel-based fiber-optic sensors for acoustic detection of partial discharges - a proof of concept," IEEE Transactions on Power Delivery, 25(4): 2526-2534, 2010.

[11] O. Kilic, M. J. F. Digonnet, G. S. Kino, and O. Solgaard, "Miniature photonic-crystal hydrophone optimized for ocean acoustics," Journal of the Acoustical Society of America, 129(4): 1837-1850, 2011.

[12] N. Lagakos, T. R. Hickman, P. Ehrenfeuchter, J. A. Bucaro, and A. Dandridge, "Planar flexible fiber-optic acoustic sensors," Journal of Lightwave Technology, 8(9): 1298-1303, 1990.

[13] G. B. Hocker, "Fiber optic acoustic sensors with increased sensitivity by use of composite structures," Optics Letters, 4(10): 320-321, 1979.

[14] G. B. Hocker, "Fiber optic acoustic sensors with composite structure: an analysis," Applied Optics, 18(21): 3679-3683, 1979.

[15] M. Imai, T. Ohashi, and Y. Ohtsuka, "Fiber-optic Michelson interferometer using an optical power divider," Optics Letters, 5(10): 418-420, 1980.

[16] M. Imai, T. Ohashi, and Y. Ohtsuka, "High-sensitive all-fiber Michelson interferometer by use of differential output configuration," Optics Communications, 39(1-2): 7-10, 1981.

[17] E. Udd, "Fibre-optic acoustic sensor based on the Sagnac interferometer," in Proc. of SPIE, vol. 425, pp. 90-95, 1983.

[18] B. J. Vakoc, M. J. F. Digonnet, and G. S. Kino, "A novel fiber-optic sensor array based on the Sagnac interferometer," Journal of Lightwave Technology, 17(11): 2316-2326, 1999.

[19] K. A. Murphy, M. F. Gunther, A. M. Vengsarkar, and R. O. Claus, "Quadrature phase-shifted, extrinsic Fabry-Pérot optical fiber sensors," Optics Letters, 16(4): 273-275, 1991.

[20] F. Xu, D. Ren, X. Shi, C. Li, W. Lu, L. Lu, et al., "High-sensitivity Fabry-Pérot interferometric pressure sensor based on a nanothick silver diaphragm," Optics Letters, 37(2): 133-135, 2012.

[21] J. Ma, W. Jin, H. L. Ho, and J. Y. Dai, "High-sensitivity fiber-tip pressure sensor with graphene diaphragm", Optics Letters, 37(13): 2493-2495, 2012.

[22] J. Ma, H. Xuan, H. L. Ho, W. Jin, Y. Yang, and S. Fan, "Fiber-optic Fabry-Pérot acoustic sensor with multilayer graphene diaphragm," IEEE Photonics Technology Letters, 25(10): 932-935, 2013. 
[23] R. E. Silva and A. A. P. Pohl, "Characterization of flexural acoustic waves in optical fibers using an extrinsic Fabry-Pérot interferometer," Measurement Science and Technology, 23(5): 055206, 2012.

[24] J. Chen, W. Li, H. Jiang, and Z. Li, "Stabilization of a fiber Fabry-Pérot interferometric acoustic wave sensor," Optik, 124(4): 339-342, 2013.

[25] X. Zou, N. Wu, Y. Tian, Y. Zhang, J. Fitek, M. Maffeo, et. al., "Ultrafast Fabry-Pérot fiber-optic pressure sensors for multimedia blast event measurements," Applied Optics, 52(6): 1248-1254, 2013.

[26] Q. Wang and Z. Ma, "Feedback-stabilized interrogation technique for optical Fabry-Pérot acoustic sensor using a tunable fiber laser," Optics \& Laser Technology, 51: 43-46, 2013.

[27] Q. Yu and X. Zhou, "Pressure sensor based on the fiber-optic extrinsic Fabry-Pérot interferometer," Photonic Sensors, 1(1): 72-73, 2011.

[28] J. H. Cole, C. Kirkendall, A. Dandridge, G. Cogdell, and T. G. Giallirenzi, "Twenty-five years of interferometric fiber optic acoustic sensors at the naval research laboratory," Washington Academy of Sciences, 90(3): 40-57, 2004.

[29] B. H. Lee, Y. H. Kim, K. S. Park, J. B. Eom, M. J. Kim, B. S. Rho, et al., "Interferometric fiber optic sensors," Sensors, 12: 2467-2486, 2012.

[30] K. O. Hill, Y. Fujii, D. C. Johnson, and B. S. Kawasaki, "Photosensitivity in optical fiber waveguides: application to reflection filter fabrication," Applied Physics Letters, 32(10): 647-649, 1978.

[31] D. J. Webb, J. Surowiec, M. Sweeney, D. A. Jackson, L. Gavrilov, J. W. Hand, et al., "Miniature fiber-optic ultrasonic probe," in Proc. of SPIE, vol. 2839, pp. 76-80, 1996.

[32] D. C. Betz, G. Thursby, B. Culshaw, and W. Staszewski, "Acousto-ultrasonic sensing using fiber Bragg gratings," Smart Materials and Structures, 12(1): 122-128, 2003.

[33] D. C. Betz, G. Thursby, B. Culshaw, and W. Staszewski, "Identification of structural damage using multifunctional Bragg grating sensors: I. theory and implementation," Smart Materials and Structures, 15(5): 1305-1312, 2006.

[34] D. C. Betz, W. Staszewski, G. Thursby, and B. Culshaw, "Structural damage identification using multifunctional Bragg grating sensors: II. damage detection results and analysis," Smart Materials and Structures, 15(5): 1313-1322, 2006.

[35] A. Cusano, A. Cutolo, J. Nasser, M. Giordano, and A. Calabro, "Dynamic strain measurements by fibre Bragg grating sensor," Sensors and Actuators A: Physical, 110(1-3): 276-281, 2004.
[36] A. Minardo, A. Cusano, R. Bernini, L. Zeni, and M. Giordano, "Response of fiber Bragg gratings to longitudinal ultrasonic waves," IEEE Transactions on Ultrasonics, Ferroelectrics and Frequency Control, 52(2): 304-312, 2005.

[37] V. Italia, A. Cusano, S. Campopiano, A. Cutolo, and M. Giordano, "Analysis of the phase response of fiber Bragg gratings to longitudinal ultrasonic fields in the high frequency regime: towards new interrogation strategies," in Proc. IEEE/LEOS Workshop on Fibers and Optical Passive Components, June 22-24, pp. 389-392, 2005.

[38] D. C. Seo, D. J. Yoon, I. B. Kwon, and S. S. Lee, "Sensitivity enhancement of fiber optic FBG sensor for acoustic emission," in Proc. of SPIE, vol. 7294, pp. $729415,2009$.

[39] G. Wild and S. Hinckley, "Optical fibre Bragg gratings for acoustic sensors," in Proc. 20th International Congress on Acoustics, Australia, August 23-27, 2010.

[40] J. A. Bucaro, N. Lagakos, B. H. Houston, S. Dey, and M. Zalalutdinov, "Compact directional acoustic sensor using a multi-fiber optical probe," The Journal of the Acoustical Society America, 133(2): 832-841, 2013.

[41] J. A. Bucaro, N. Lagakos, B. H. Houston, J. Jarzynski, and M. Zalalutdinov, "Miniature, high performance, low cost fiber optic microphone," The Journal of the Acoustical Society America, 118(3): 1406-1413, 2005.

[42] G. He and F. W. Cuomo, "Displacement response, detection limit, and dynamic range of fiber-optic level sensors," Journal of Lightwave Technology, 9(11): 1618-1625, 1991.

[43] F. Yang, W. Jin, H. L. Ho, F. Wang, W. Liu, L. Ma, et al., "Enhancement of acoustic sensitivity of hollow-core photonic bandgap fibers," Optics Express, 21(13): 15514-15521, 2013.

[44] J. H. Cole, C. Sunderman, A. B. Tveten, C. Kirkendall, and A. Dandridge, "Preliminary investigation of air-included polymer coatings for enhanced sensitivity of fiber-optic acoustic sensors," in Proc. of 15th International Conference on Optical Fiber Sensors, Portland, USA, May 10, vol. 1, pp. 317-320, 2002.

[45] J. H. Cole, S. Mothley, J. Jarzynski, A. B. Tveten, C. Kirkendall, and A. Dandridge, "Air-included polymer coatings for enhanced sensitivity of fiber-optic acoustic sensors," in Proc. of 16th International Conference on Optical Fiber Sensors, Nara, Japan, pp. 214-217, 2003.

[46] S. E. U. Lima, R. G. Farias, F. M. Araújo, L. A. Ferreira, J. L. Santos, V. Miranda, et al., "Fiber laser sensor based on a phase-shifted chirped grating for 
acoustic sensing of partial discharges," Photonic Sensors, 3(1): 44-51, 2013.

[47] A. I. Azmi, D. Sen, W. Sheng, J. Canning, and G. D. Peng, "Performance enhancement of vibration sensing employing multiple phase-shifted fiber Bragg grating," Journal of Lightwave Technology, 29(22): 3453-3460, 2011.

[48] A. I. Azmi, Raju, and G. D. Peng, "Failure monitoring of e-glass/vinylester composites using fiber grating acoustic sensors," Photonic Sensors, 3(2): 184-192, 2013.

[49] Z. Qi, H. Huang, T. Cao, P. Liu, Z. Tang, and B. Qu "Highly sensitive fiber pressure sensor based on off-center diaphragm reflection," Applied Optics, 52(18): 4223-4227, 2013.

[50] M. J. F. Digonnet, O. C. Akkaya, G. S. Kino, and O. Solgaard, "Miniature fiber acoustic sensors and sensor array using photonic-crystal membranes," in Imaging and Applied Optics Technical Papers, OSA Technical Digest, California, USA, June 24-28, pp. stu3f.1, 2012.
[51] W. Suh, M. F. Yanik, O. Solgaard, and S. H. Fan, "Displacement-sensitive photonic crystal structures based on guided resonance in photonic crystal slabs," Applied Physics Letters, 82(13): 1999-2001, 2003.

[52] Y. Li, X. Wang, and X. Bao, "Sensitive acoustic vibration sensor using single-mode fiber tapers," Applied Optics, 50(13): 1873-1878, 2011.

[53] R. Chen, G. F. Fernando, T. Butler, and R. A. Badcock, "A novel ultrasound fibre optic sensor based on a fused-tapered optical fibre coupler," Measurement Science and Technology, 15: 1490-1495, 2004.

[54] M. S. Ferreira, M. Becker, H. Bartelt, P. Mergo, J. L. Santos, and O. Frazão, "A vibration sensor based on a distributed Bragg reflector fibre laser," Laser Physics Letters, 10(9): 095102, 2013.

[55] M. S. Ferreira, J. L. Santos, P. Mergo, and O. Frazão, "Torsion sensor based on a figure-of-eight cavity fibre laser," Laser Physics Letters, 10(4): 045105, 2013. 